\title{
Quasinormal modes of black holes. II. Padé summation of the higher-order WKB terms.
}

\author{
Jerzy Matyjasek ${ }^{1, *}$ and Małgorzata Telecka ${ }^{2}$ \\ ${ }^{1}$ Institute of Physics, Maria Curie-Sktodowska University \\ pl. Marii Curie-Sktodowskiej 1, 20-031 Lublin, Poland \\ ${ }^{2}$ Faculty of Earth Sciences and Spatial Management, \\ Maria Curie-Sktodowska University, \\ Al. Kraśnicka 2cd, 20-031 Lublin, Poland
}

\begin{abstract}
In previous work [1] we proposed an improvement of the WKB-based semianalytic technique of Iyer and Will for calculation of the quasiormal modes of black holes by constructing the Padé approximants of the formal series for $\omega^{2}$. It has been demonstrated that (within the domain of applicability) the diagonal Padé transforms $\mathcal{P}_{6}^{6}$ and $\mathcal{P}_{7}^{6}$ are always in a very good agreement with the numerical results. In this paper we present a further extension of the method. We show that it is possible to reproduce many known numerical results with a great accuracy (or even exactly) if the Padé transforms are constructed from the perturbative series of a really high order. In our calculations the order depends on the problem but it never exceeds 700. For example, the frequencies of the gravitational mode $l=2, n=0$ calculated with the aid of the Padé approximants and within the framework of the continued fractions method agree to 24 decimal places. The use of such a large number of terms is necessary as the stabilization of the quasinormal frequencies can be slow. Our results reveal some unexpected features of the WKB-based approximations and may shed some fresh light on the problem of overtones.
\end{abstract}

\section{INTRODUCTION}

The quasinormal modes of black holes [2] have been the subject of intense studies over the last 40 years and the reason for this continuous interest stems from the fact that they were expected to be detectable. Indeed, a perturbed black hole undergoes the ringdown phase in which the oscillations are characterized by a set of complex frequencies, $\omega$. The real part of each $\omega$ gives the oscillation frequency whereas the imaginary part determines the characteristic damping rate and consequently the quasinormal modes are crucial in detecting and subsequently studying the prop-

*Electronic address: jurek@kft.umcs.lublin.pl, jirinek@gmail.com 
erties of the gravitational waves generated in the violent collisions of black holes or gravitational collapse. Because of the constant and still growing interest in the quasinormal modes many numerical and analytical approaches have been proposed for their calculation. The most popular are the highly accurate numerical methods, such as the method of continued fractions [3 5] constructed by applying the Frobenius series solution, asymptotic iteration [6], the method of the Hill determinant [7, the method of Nollert and Schmidt [8] and the pseudospectral method [9]. Recently, in a very interesting development [10, 11], the quasinormal modes of the parametrized black holes have been studied. On the opposite side we have a bunch of the approximate analytic and semianalytic methods mainly based on the WKB expansion and its variants [12 18], the perturbative approach of Ref. [19] and the approach advocated by Gal'tsov and Matiukhin [20]. Especially interesting is the method developed by Zaslavskii [19], who following ideas of Refs. [21 23] reduced the problem to the quantum anharmonic oscillator and used powerful Rayleigh-Schrödinger perturbation theory.

We assume that the second order differential equation describing perturbations (with a time dependence of the form $e^{-\omega t}$ ) can be reduced to the Schrödinger-type equation

$$
\left[\frac{d^{2}}{d x^{2}}+Q(x)\right] \psi(x)=0
$$

where $Q(x)=\omega^{2}-V(x), V(x)$ is a potential and $x$ is the tortoise coordinate. The potential is constant as $|x| \rightarrow \infty\left(V(-\infty)\right.$ and $V(\infty)$ may be different) and has maximum at $x_{0}$. The radial part of the free oscillations are described by the functions $\psi(x)$ which are purely "outgoing" as $|x| \rightarrow \infty$, i.e., the functions that are moving away from the potential barrier in both directions. For a perturbation of a given spin weight, $s$, the quasinormal modes are labeled by the multipole number, $l$, and the overtone number $n$.

Typically, the form of the potential function practically excludes the possibility of finding an exact solution of Eq.(1) and consequently one is forced to adopt the numerical or approximate methods. One of the most popular approximate (semianalytic) methods is the Iyer-Schutz-Will approach [12, 13] and its generalization to the sixth-order WKB [24]. The Iyer-Schutz-Will modification of the WKB method in its original form allows for potentials with closely lying classical turning points. It can be achieved by constructing the approximate solution in the region between the turning points, which is expressible in terms of the parabolic cylinder function, and matching them with the approximate WKB solutions in the exterior regions. For example, in Ref. [13] the third order WKB solutions are asymptotically matched with the solutions constructed for the 6th-order Taylor approximation of the potential. It is interesting that the results presented in [13. 
have been reconstructed by Zaslavskii, who used textbook formulas describing the energy levels of the anharmonic oscillator and by Galt'sov and Matukhin. The Iyer-Schutz-Will method has been subsequently extended to higher order in Ref. [24].

A natural question that arises in this context is whether one could construct a better approximation yielding more accurate results. In Ref. [1] (henceforth Paper I) it was shown that the answer to this question is affirmative. The strategy adopted in that paper is to extend the order of the WKB terms (and consequently the order of the Taylor series approximation of the potential) and construct the diagonal Padé approximants of the formal series for $\omega^{2}$. It has been shown that within the domain of validity of the method, the diagonal Padé transforms $\mathcal{P}_{6}^{6}$ and $\mathcal{P}_{7}^{6}$ are always in excellent agreement with the numerical (exact) results constructed for the perturbed Schwarzschild and Reissner-Nordström black holes. Moreover, it has been shown that the method works well even for the fundamental low-lying modes of the perturbed Tangherlini black hole. Unfortunately the analytic calculations of the higher order terms of the WKB approximation are very complicated and each next order requires more and more time. Recently, in a calculation lasting tens of hours, we have calculated all the terms up to 16 th order, which allow to extend our method to $\mathcal{P}_{8}^{8}$ transforms. However, it would be interesting to extend and verify our method in the regime of higher order WKB. There is little hope that this can be done analytically for a general potential as the number of terms as well as their complexity grows fast with the order. On the other hand, however, one expects that there should be massive simplifications when the calculations are performed for a given potential with prescribed multipole and overtone numbers. Another way out of the impasse would be the extension of Zaslavskii's approach to really high orders. Recently, in the interesting and important paper devoted to Borel summation of the analytically continued Padé transforms, Hatsuda 25] propounded to make use of the efficient Mathematica package written by Sulejmanpasic and Ünsal [26] (see also Ref. 27] and the references therein). This package allows calculation of the energy corrections of the one-dimensional system with an arbitrary locally-harmonic potential. This, in turn, leads us to the method advocated by Zaslavskii.

In this paper we report on a further extension of our method by using a combination of different procedures, which will reveal some unexpected features of the WKB approximation and will shed fresh light on the problem of overtones. The main emphasis is put on accuracy that can be achieved using relatively simple techniques and, as in Paper I, we are looking for a simple, WKB-based approximation that can be used, practically without any changes, for a wide class of metrics. This black-box nature of the method, with the effective potential taken as the input and the accurate frequencies of the the quasinormal modes obtained as the output, should be considered as its great 
TABLE I: The number of terms in $\Lambda_{k}$ for $2 \leq k \leq 16$.

\begin{tabular}{cccccccccccccccc}
\hline \hline$\Lambda_{n}$ & $\Lambda_{2}$ & $\Lambda_{3}$ & $\Lambda_{4}$ & $\Lambda_{5}$ & $\Lambda_{6}$ & $\Lambda_{7}$ & $\Lambda_{8}$ & $\Lambda_{9}$ & $\Lambda_{10}$ & $\Lambda_{11}$ & $\Lambda_{12}$ & $\Lambda_{13}$ & $\Lambda_{14}$ & $\Lambda_{15}$ & $\Lambda_{16}$ \\
\hline Number of terms & 6 & 20 & 55 & 132 & 294 & 616 & 1215 & 2310 & 4235 & 7524 & 13026 & 22050 & 36540 & 59488 & 95268 \\
\hline \hline
\end{tabular}

advantage. The results presented in this paper raise the question of the limitations of the method. The usual interpretation is that the method works well for low overtones and the approximation gets progressively better with increasing $l$. However, the results presented in this paper show that one can achieve great accuracy even for higher overtones at the expense of increasing of the order of the perturbation series.

\section{THE METHOD}

Although the derivation of the equations of the standard Iyer-Schutz-Will method is rather complicated it leads, surprisingly, to quite simple final result. Since the method and its generalizations have been presented in details in Refs. [1, 12, 13] we shall omit most of its technical aspects here.

The formula relating the complex frequencies of the quasinormal modes and the derivatives of $Q(x)$ at $x=x_{0}$ can be written in a compact form $(n=0,1,2, \ldots)$

$$
\frac{i Q_{0}}{\sqrt{2 Q_{0}^{\prime \prime}} \varepsilon}-\sum_{k=2}^{N} \varepsilon^{k-1} \Lambda_{k}=n+\frac{1}{2}
$$

where $x_{0}$ is a point at which the potential has its maximum, each $\Lambda_{k}$ is combination of the derivatives of $Q(x)$ calculated at $x_{0}$ and $\varepsilon$ is the expansion parameter that helps to keep track of the order of terms. The complexity of $\Lambda_{k}$ terms increases with $k$ and the number of the constituents for $k \leq 16$ is given in Table $\mathrm{I}$.

Now, let us assume that the potential does not depend on $\omega$. In a standard approach one puts $\varepsilon=1$ and for a given potential solves Eq. 2 with respect to $\omega$. That means that the $\Lambda_{k}$ terms are summed. On the other hand, however, we do not know in advance if taking into account additional $\Lambda$ terms will improve the quality of the results. Moreover, one cannot exclude the possibility that the improvement is just accidental. To remedy this problem it has been proposed to treat the expression for the quasinormal frequencies as the formal perturbation expansion

$$
\omega^{2}=V\left(x_{0}\right)-i\left(n+\frac{1}{2}\right) \sqrt{2 Q_{0}^{\prime \prime}} \varepsilon-i \sqrt{2 Q_{0}^{\prime \prime}} \sum_{i=2}^{N} \varepsilon^{j} \Lambda_{j} \equiv V\left(x_{0}\right)+\sum_{i=1}^{N} \varepsilon^{i} \tilde{\Lambda}_{i}
$$


and instead of summing the terms in the right hand side of (3) (which is a bad strategy) to construct the Padé approximants [1]. As is well known the Padé approximants associated with a formal power series $\sum a_{k} x^{k}$ are defined as the unique rational functions $\mathcal{P}_{N}^{M}(x)$ of degree $N$ in the denominator and $M$ in the numerator satisfying

$$
\mathcal{P}_{N}^{M}(x)-\sum_{k=0}^{M+N} a_{k} x^{k}=\mathcal{O}\left(x^{M+N+1}\right)
$$

In our previous paper we have extended the WKB-based calculations to the 13th-order, i.e., we have calculated all $\Lambda_{k}$ up to $k=13$ and for any given multipole $l$ and the overtone number $n$ we used the Padé summation [28]. It has been demonstrated that for the so-far best documented quasinormal frequencies of the Schwarzschild and Reissner-Nordström black holes the diagonal Padé transforms $\mathcal{P}_{6}^{6}$ and $\mathcal{P}_{7}^{6}$ are always in very good agreement with the exact numerical results. Of course the approximation has its own limitations mainly related to the limitations of the WKB theory. On the other hand, the deviations of the complex frequencies obtained within the framework of the improved method from the numerical results are smaller than the analogous results obtained with the aid of competing (approximate) approaches. In this paper we extend the analyses of Ref. [1] in two ways: first we will construct the $\Lambda_{k}$ functions for $k \leq 16$ and subsequently use the Wynn epsilon algorithm for convergence acceleration [29, 30]. Details of the algorithm are relegated to Appendix. Although the formulas describing $\Lambda_{k}$ are rather complicated the calculations can be substantially accelerated by the choice of the suitable strategy. As the construction of the functions $\Lambda_{k}$ has been described in detail in Refs. [1, 12, 13] we will not repeat it here. Unfortunately, if one is interested in really high orders of the WKB approximation the analytic approach briefly described above is hard, if not impossible, to use. Although its numerical variant would certainly simplify and speed up the calculations here we will follow a different path. We will make use of the Zaslavskii approach in which the resonant scattering problem is converted into the bound state problem of the anharmonic oscillator. The simplest method to do so is to exploit a formal equivalence between the master equation (1) with the function $Q(x)$ expanded in a Taylor series about the point $x_{0}$ at which it has a maximum and the equation that describes quantum-mechanical anharmonic oscillator. Indeed, reducing the latter problem to the simple application of the standard perturbation theory, one has

$$
\left(H_{0}+\tilde{V}\right) \psi=E \psi
$$

where

$$
H_{0}=-\frac{1}{2} \frac{d^{2}}{d x^{2}}+\frac{1}{4} Q_{0}^{(2)} x^{2}
$$




$$
\tilde{V}=\frac{1}{2} \sum_{k=3}^{N} \varepsilon^{\frac{k}{2}-1} Q_{0}^{(k)} \frac{x^{k}}{k !}
$$

and $Q_{0}^{(k)}$ is $k$-th derivative of $Q(x)$ at $x_{0}$. Now, calculating the perturbative corrections to the energy levels, multiplying the thus obtained results by the factor $\sqrt{2 / Q_{0}^{(2)}}$ and finally making the substitution $\varepsilon \rightarrow i \varepsilon$ one obtains precisely the $\Lambda_{j}$ functions of Eq. (3).

As have been observed by Zaslavskii, the first two nontrivial correction terms, i.e. the Iyer-Will result, can easily be calculated using almost entirely textbook results [31]. Unfortunately, the textbook-based method is rather inefficient in the higher-order calculations. Recently, in a very interesting paper Sulejmanpasic and and Ünsal [26] extended the method developed by Bender and $\mathrm{Wu}$ [27] (and originally applied to the simple anharmonic oscillator) to the arbitrary locally harmonic potential. The paper is accompanied with the very efficient computer algebra package, allowing construction of the really high orders of the perturbation expansion. Although the practical applicability of the package for the analytic calculations of the general functions $\Lambda_{k}$ is limited to first few orders its real power lies in the numerics ${ }^{1}$ Our strategy is as follows: First we calculate the complex frequencies of the black hole normal modes using the general $\Lambda_{k}(k \leq 16)$ constructed within the framework of the Iyer-Will approach and subsequently, for a given harmonic and overtone numbers, we calculate numerically the high orders of parameters $\Lambda_{k}$. (Note the slightly abused notation). The choice of the maximal $k$ used in this paper depends on the type of the modes, but it never exceeds $k=700$. Having computed all the necessary $\Lambda_{k}$ we construct the Padé approximants of Eq. (3) treated as a formal polynomial of $\varepsilon$. We show that one can obtain amazingly accurate results even in the situations typically considered as too hard for the WKB-based methods.

We conclude this section with a few observations on the approach proposed in Ref. [25]. First it should be observed that although the Padé transforms, $\tilde{\mathcal{P}}_{N}^{N}$ of the series $\sum_{k} a_{k} x^{k} / k$ ! can easily be constructed, the calculation of the integral transform of $\tilde{\mathcal{P}}_{N}^{N}$, denoted here by $\mathcal{B}_{N}^{N}$

$$
\mathcal{B}_{N}^{N}=\int_{0}^{\infty} e^{-\xi} \tilde{\mathcal{P}}_{N}^{N}(\xi x) d \xi
$$

could be time consuming, especially for $k>100$. Moreover, the calculations of $\mathcal{B}_{N}^{N}$ for $k>100$ suffer (at least in our implementation of the algorithm) from numerical instabilities, and, consequently, one has to be very careful in choosing the calculational strategy and in interpreting the thus obtained results.

\footnotetext{
${ }^{1}$ We have checked that for $k \leq 5$ the $\Lambda_{k}$ calculated with the aid of the BenderWu package are precisely the same as the functions $\Lambda_{k}$ constructed within the framework of the Iyer-Will approach. The equality of the results for $6 \leq k \leq 16$ has been verified numerically.
} 


\section{QUASINORMAL MODES}

\section{A. The Schwarzschild black hole}

In this section we shall construct the quasinormal modes of the perturbed Schwarzschild black hole. Although the Padé summation is our method of choice, whenever in doubt, we will also use the Borel-Padé summation [25]. Moreover, all the cases presented in this section have been calculated using the continued fraction method. It should be stressed that the Padé transforms work extremely well and our results are in perfect agreement with the continued fraction method. We have also corrected a few erroneous results, which have appeared in the literature.

First let us consider the odd-parity (Regge-Wheeler) potential

$$
V\left(r_{*}\right)=\left(1-\frac{1}{r}\right)\left(\frac{L}{r^{2}}+\frac{1-s^{2}}{r^{3}}\right)
$$

where $L=l(l+1)$ and $s=0,1$ or 2 for the scalar, vector and gravitational perturbations, respectively. The problem of construction of the complex frequencies of the quasinormal modes reduces to a simple application of the formula (3). The results of our calculations are tabulated in Tables II XIII and the accurate numerical results presented at the top of each column are taken from Ref. [32]. Generally speaking all the results follow the same pattern which is clearly visible in Figs. 1 and 2, Each point represent the real (Fig,1) or imaginary (Fig 2) part of the complex frequency $\omega$ constructed for a given $\mathcal{P}_{k}^{k}$ for a $s=2, l=2$ and $n=7$. Inspection of the Figures shows that even for $k=100$ the spread of $\omega$ is quite big, and that the result seems to stabilize starting with $k=150$. The horizontal line represents the exact numerical result. The analogous behavior of lower overtones is similar to that discussed above with the one reservation: the spread of $\omega$ is smaller and stabilization starts for smaller values of $k$. The lesson that follows from this test case is that one should be cautious with accepting the results based on only a few first $\Lambda$ terms. Similarly, the equality of a few consecutive results does not necessarily mean that the final stable result is approached. The above discussion raises two questions: Does this pattern hold for higher overtones, and is it possible to reconstruct the algebraically special modes this way. Although a complete answer to this questions is beyond our understanding, we can, nevertheless, make some observations. First, it seems impossible to construct the algebraically special modes, say the gravitational mode $(2,8)$, by any of the WKB-based methods. For an interesting discussion see Andersson's paper [32]. The rest of this section is devoted to the first question.

The common wisdom regarding the applicability of the WKB method to the quasinormal mode problem is that it gives, at best, only approximate results, satisfactorily reproduces only the fun- 
damental modes and works really great for $l \gg 1$. Moreover, the frequencies of the overtones can be calculated with some confidence only for $n \lesssim l$ and rapidly deteriorates with the index number. And finally, summing the first few terms of the perturbative series for $\omega^{2}$ can give more accurate results. Below we shall show that some of the above limitations may be weakened or even overcome except the last point which is generally untrue.

To analyze the quality of the approximation in more details it is convenient to define deviation of the real part of the frequency

$$
\Delta^{(r)}\left(\omega_{k}\right)=\frac{\Re\left(\omega_{k}\right)-\Re\left(\omega_{\text {num }}\right)}{\Re\left(\omega_{\text {num }}\right)} 100 \%
$$

and the deviation of its imaginary part

$$
\Delta^{(i)}\left(\omega_{k}\right)=\frac{\Im\left(\omega_{k}\right)-\Im\left(\omega_{\text {num }}\right)}{\Im\left(\omega_{\text {num }}\right)} 100 \%,
$$

where $\omega_{k}$ is the approximate complex frequency of the quasinormal mode and $\omega_{\text {num }}$ is its accurate numerical value. Now, consider for example the scalar (odd) $(0,10)$ mode. Our calculations show that the deviations of $\omega$ constructed from $\mathcal{P}_{350}^{350}$ are $\Delta^{(r)}(\omega)=6.37 \times 10^{-2 \%}$ and $\Delta^{(i)}(\omega)=6.14 \times$ $10^{-4 \%}$, respectively. Although the accuracy of this result is quite good, and we expect that for the higher-order transforms it would be even better, the calculation becomes progressively more difficult, and, consequently, there is a natural limit of applicability of the method ${ }^{2}$. On the other hand, for a given order of the Padé sum the accuracy of the approximation increases with the harmonic index $l$ and deteriorates with $n$.

To simplify our discussion we shall denote $l, n$ mode by $(l, n)$ and the Borel-Padé sum constructed from the diagonal Padé transform $\tilde{\mathcal{P}}_{N}^{N}$ by $\mathcal{B}_{N}^{N}$. Since the numerical calculations reported in Ref. [32] were limited to an accuracy of nine decimal places (with the exception of the scalar $(0,0),(0,1),(1,0)$ and $(1,1)$ modes) our results, for comparison, have been rounded-off accordingly. (In the tables the results of Ref. [32] have been multiplied by 2, whereas for numerical comparison our results have been divided by 2 ).

First, let us consider the lowest scalar mode $(0,0)$. This mode is somewhat mysterious as there is a discrepancy between the exact numerical results and the results obtained within the framework of the Padé summation (see Tab. II). The frequency calculated by Andersson is 0.2209086-0.2097886i, whereas the Padé summation gives the result that differs at the 6th and 5th decimal places for the real and imaginary part of $\omega$, respectively. Moreover, to the order considered in this paper, the

\footnotetext{
${ }^{2}$ The total time of calculations rapidly grows with $k$.
} 


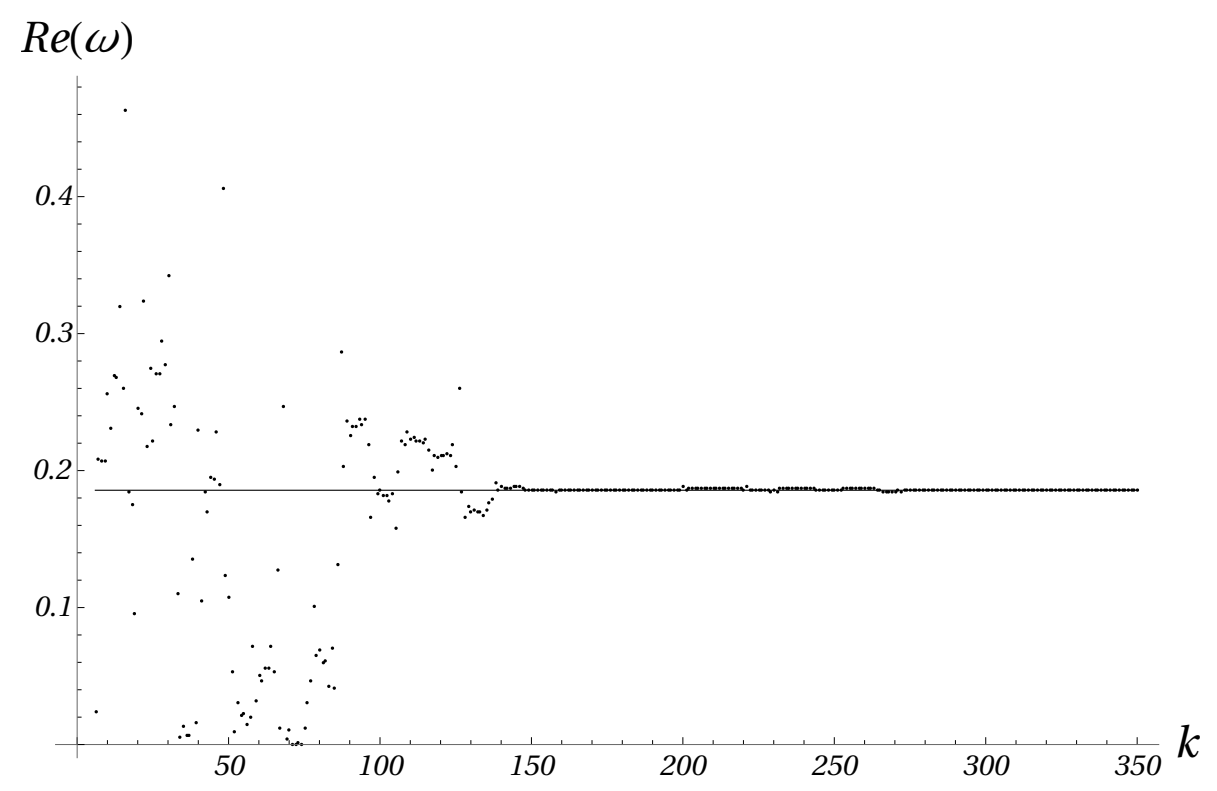

FIG. 1: The real part of the quasinormal frequency of the odd gravitational perturbation with $l=2, n=7$ calculated for 345 Padé transforms $\mathcal{P}_{k}^{k}, 6 \leq k \leq 350$. The horizontal thin solid line represents the exact numerical result.

result seems to stabilize with the rather high accuracy of 16 decimal places. Additionally we have calculated the complex frequency of $(0,0)$ mode using the Borel-Padé summation and for $\mathcal{B}_{249}^{249}$ and $\mathcal{B}_{250}^{250}$ the results obtained are in concord with the Padé transforms rather than the result presented in Ref. [32. Indeed, the results obtained within the framework of the both methods agree to 12 decimal places. It is possible that the WKB-based methods give the convergent result that differs form the exact numerical one. On the other hand however, it can be seen from Tab. III that for the mode $(0,1)$ one has $\Delta^{(r)}=\Delta^{(i)}=0$ and the complex frequencies of the higher overtones are amazingly accurate (Tabs. IV VII). To solve this problem we have calculated $\omega$ using the method of continued fraction with the Wynn algorithm to accelerate convergence of the approximants. Our code (a very unsophisticated one) gives for the fundamental frequency precisely the same result as the Padé summation to the accuracy quoted, i.e., the results are identical to 16 decimal places. This equality, although expected, is really impressive, especially taking into account the deep differences in the computational strategies. For a better confidence we have computed the frequency of this mode without the series acceleration in the continued fraction method and obtained precisely the same result. There is also a small discrepancy in the real part of the scalar mode $(1,0)$. Inspection of Tab. II shows that we have precisely the same situation as the one discussed above. We believe that our results are correct. 


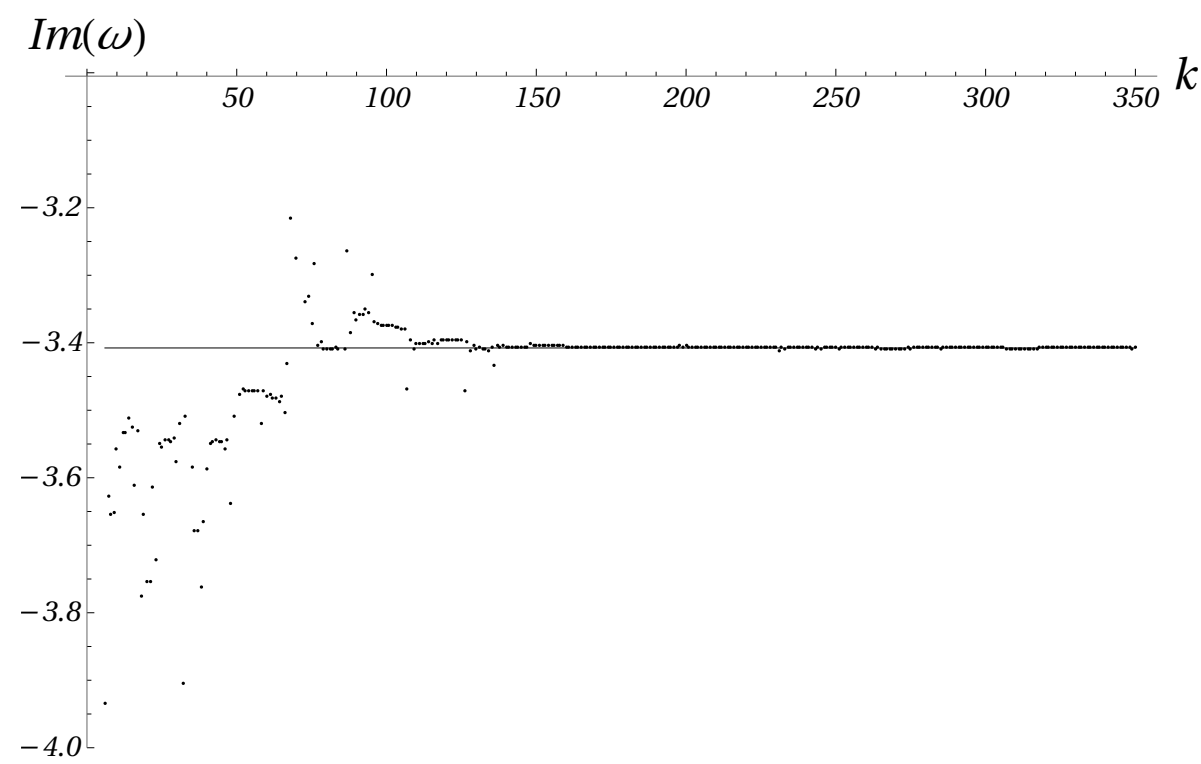

FIG. 2: The imaginary part of the quasinormal frequency of the odd gravitational perturbation with $l=2$, $n=7$ calculated for 345 Padé transforms $\mathcal{P}_{k}^{k}, 6 \leq k \leq 350$. The horizontal thin solid line represents the exact numerical result.

It should be emphasized that although the results are tabulated only for a few exemplary values of $k$ we have calculated $\omega$ for all $k$ satisfying $6 \leq k \leq k_{\max }$, where $k_{\max }$ is the maximal order of the Padé transform involved in the calculations. That, in turn, means that determination of the Padé transform $\mathcal{P}_{k}^{k}$ requires knowledge of the perturbation series to the order $2 k$. Now, let us consider the scalar modes $l=1$ listed in the right columns of Tabs. II [VII. Our calculations show that the Padé approximants yield frequencies that are in a perfect agreement $\left(\Delta^{(r)}=\Delta^{(i)}=0\right)$ with the numerical calculations reported in Ref. [32].

The Padé approximants for the electromagnetic modes are also accurate. Indeed, the deviations of the vector $l=1$ modes (see Tables VIII XIII) are identically zero for all considered cases. It should be noted however, that the result for the scalar $(1,0)$ mode (see Tab. VIII) presented in Ref. [32] differs from our calculations and to establish which result is the correct one we have compared the frequency with the result obtained using the continued fraction method. Inspection of the table shows that the Padé transforms and the continued fraction method give precisely the same $\omega$, and, consequently, we believe that our result is correct.

Finally, for the gravitational modes listed in the right columns of Tabs. VIII,XIII, one finds $\left|\Delta^{(r)}\right| \propto 10^{-7} \%$ and $\left|\Delta^{(r)}\right| \propto 10^{-6} \%$ for $n=4$ and $n=5$, respectively. Similarly, for the deviations of the imaginary parts one has $\left|\Delta^{(i)}\right|=\propto 10^{-6} \%$ for $n=4$ and $n=5$. The remaining results exactly reproduce the numerically calculated frequencies. 
TABLE II: The complex frequencies of the scalar quasinormal modes for $s=0, l=0, n=0$ (left column) and $s=0, l=1, n=0$ (right column). The Padé approximants, $\mathcal{P}_{k}^{k}$, are calculated for $k=$ $6,8,50,100,150,200,250$ and 300 . The last row is calculated within the framework of the continued fraction method.

\begin{tabular}{rcc}
\multicolumn{1}{c}{$l=0, n=0$} & $l=1, n=0$ \\
\hline & $0.2209086-0.2097886 i$ & $0.5858722-0.19532 i$ \\
\hline 6 & $0.2226575720860749-0.2097705214916321 i$ & $0.5858723081529635-0.1953200537758777 i$ \\
8 & $0.2209467125923617-0.2099078086678199 i$ & $0.5858722675722460-0.1953199825012170 i$ \\
50 & $0.2209098826757620-0.2097914422244537 i$ & $0.5858722665345654-0.1953199778271564 i$ \\
100 & $0.2209098781648214-0.2097914341697581 i$ & $0.5858722665345654-0.1953199778271564 i$ \\
150 & $0.2209098781608154-0.2097914341737470 i$ & $0.5858722665345654-0.1953199778271564 i$ \\
200 & $0.2209098781608393-0.2097914341737620 i$ & $0.5858722665345654-0.1953199778271564 i$ \\
250 & $0.2209098781608394-0.2097914341737619 i$ & $0.5858722665345654-0.1953199778271564 i$ \\
300 & $0.2209098781608394-0.2097914341737619 i$ & $0.5858722665345654-0.1953199778271564 i$ \\
$\mathrm{CF}$ & $0.2209098781608394-0.2097914341737619 i$ & $0.5858722665345654-0.1953199778271564 i$
\end{tabular}

TABLE III: The complex frequencies of the scalar quasinormal modes. The Padé approximants, $\mathcal{P}_{k}^{k}$, are calculated for $k=6,8,50,100,150,200$ and 250 .

\begin{tabular}{ccc}
$k$ & $l=0, n=1$ & $l=1, n=1$ \\
\hline $0.1722338-0.6961048 i$ & $0.5288973-0.61251478 i$ \\
\hline 6 & $0.1739745319353183-0.6959893881331765 i$ & $0.5288861492458066-0.6125171735439730 i$ \\
8 & $0.1757654368519873-0.6957757352543876 i$ & $0.5288974070506602-0.6125174288621156 i$ \\
50 & $0.1722542901637571-0.6961018341205277 i$ & $0.5288973012096589-0.6125147831180967 i$ \\
100 & $0.1722345014978213-0.6961051951489169 i$ & $0.5288973012096651-0.6125147831180942 i$ \\
150 & $0.1722338624300778-0.6961048943217377 i$ & $0.5288973012096651-0.6125147831180942 i$ \\
200 & $0.1722338376996682-0.6961048946120284 i$ & $0.5288973012096651-0.6125147831180942 i$ \\
250 & $0.1722338367597048-0.6961048936507108 i$ & $0.5288973012096651-0.6125147831180942 i$
\end{tabular}

We have focused on the Regge-Wheeler potential. One expects that the quality of the approximation grows with $l$. Similar calculations have been carried out for the Zerilli potential, and, as expected, the obtained results are even better. Unfortunately, the calculations for the even parity modes, because of the form of the Zerilli potential, are more complicated. As they add little to the discussion they will not be presented here. 
TABLE IV: The complex frequencies of the scalar quasinormal modes. The Padé approximants, $\mathcal{P}_{k}^{k}$, are calculated for $k=6,8,50,100,150,200$ and 250 .

\begin{tabular}{rcc}
\multicolumn{1}{c}{$l=0, n=2$} & $l=1, n=2$ \\
\hline 6 & $0.1501610934536142-1.2183646740525384 i$ & $0.4584542419793649-1.0801478608208415 i$ \\
8 & $0.1638975530635953-1.2177361392404707 i$ & $0.4588978123676309-1.0803319265755279 i$ \\
50 & $0.1525086624622595-1.2030996120120083 i$ & $0.45907866697505832-1.0802668500082639 i$ \\
100 & $0.1514835672117383-1.2021637621538090 i$ & $0.4590786698626058-1.0802668500382169 i$ \\
150 & $0.1514855070708692-1.2021579660190725 i$ & $0.4590786698626033-1.0802668500382144 i$ \\
200 & $0.1514840139830334-1.2021573800732860 i$ & $0.4590786698626033-1.0802668500382144 i$ \\
250 & $0.1514838833327049-1.2021571932998896 i$ & $0.4590786698626033-1.0802668500382144 i$
\end{tabular}

TABLE V: The complex frequencies of the scalar quasinormal modes. The Padé approximants, $\mathcal{P}_{k}^{k}$, are calculated for $k=6,8,50,100,150,200$ and 250 .

\begin{tabular}{rcc}
\hline \multicolumn{1}{c}{$l=0, n=3$} & $l=1, n=3$ \\
\hline & $0.140820276-1.707354636 i$ & $0.406516772-1.576595646 i$ \\
\hline 6 & $0.138060417273466-1.732103754671567 i$ & $0.405851086039425-1.577770124846531 i$ \\
8 & $0.137670535339324-1.731506857378750 i$ & $0.406197938547988-1.576954341222488 i$ \\
50 & $0.142414546944632-1.706681174348947 i$ & $0.406516784404676-1.576595635301799 i$ \\
100 & $0.140982067753947-1.707290791968112 i$ & $0.406516772362319-1.576595645568015 i$ \\
150 & $0.140847814828334-1.707387507328528 i$ & $0.406516772366920-1.576595645562394 i$ \\
200 & $0.140821949939888-1.707357238989158 i$ & $0.406516772366927-1.576595645562396 i$ \\
250 & $0.140820464201770-1.707355063984697 i$ & $0.406516772366927-1.576595645562396 i$ \\
\hline
\end{tabular}


TABLE VI: The complex frequencies of the scalar quasinormal modes. The Padé approximants, $\mathcal{P}_{k}^{k}$, are calculated for $k=6,8,50,100,150,200$ and 250 .

\begin{tabular}{rcc}
\hline \multicolumn{1}{c}{$l=0, n=4$} & $l=1, n=4$ \\
\hline & $0.134148608-2.21126376 i$ & $0.37021804-2.081524226 i$ \\
\hline 6 & $0.124765034276338-2.229022763903854 i$ & $0.369834774192733-2.084205198945555 i$ \\
8 & $0.131144317225359-2.239048678679388 i$ & $0.369767198720716-2.081642311885022 i$ \\
50 & $0.140769758034688-2.209616863307420 i$ & $0.370217997741403-2.081524178098750 i$ \\
100 & $0.133932901572174-2.212104617277194 i$ & $0.370218041051879-2.081524225583624 i$ \\
150 & $0.134187347043185-2.211290539046596 i$ & $0.370218040690568-2.081524225634893 i$ \\
200 & $0.134168230131617-2.211267909887815 i$ & $0.370218040690412-2.081524225635177 i$ \\
250 & $0.134149076601310-2.211261806257428 i$ & $0.370218040690404-2.081524225635138 i$ \\
\hline
\end{tabular}

TABLE VII: The complex frequencies of the scalar quasinormal modes. The Padé approximants, $\mathcal{P}_{k}^{k}$, are calculated for $k=6,8,50,100,150,200,250,300$ and 350 .

\begin{tabular}{rcc}
\hline \multicolumn{1}{c}{$l=0, n=5$} & $l=1, n=5$ \\
\hline & $0.129483444-2.71427893 i$ & $0.344153622-2.588239396 i$ \\
\hline 6 & $0.122524417275065-2.726977710003108 i$ & $0.349813936639104-2.592216963795157 i$ \\
8 & $0.127292811291479-2.746960760117273 i$ & $0.340849558720576-2.589050268997814 i$ \\
50 & $0.146613305792775-2.691720611629606 i$ & $0.344154713036727-2.588239612402011 i$ \\
100 & $0.133076394282346-2.714297398099659 i$ & $0.344153626253300-2.588239388487938 i$ \\
150 & $0.129736760525904-2.714220815430054 i$ & $0.344153622489611-2.588239396618380 i$ \\
200 & $0.129545864551587-2.714271452225676 i$ & $0.344153622500862-2.588239396622716 i$ \\
250 & $0.129501756504425-2.714283142375501 i$ & $0.344153622501171-2.588239396622020 i$ \\
300 & $0.129484950919583-2.714282779384554 i$ & $0.344153622501291-2.588239396621862 i$ \\
350 & $0.129484811363228-2.714277877939901 i$ & $0.344153622501297-2.588239396621861 i$ \\
\hline
\end{tabular}


TABLE VIII: The complex frequencies of the electromagnetic (left column) and the gravitational (right column) quasinormal modes. The Padé approximants, $\mathcal{P}_{k}^{k}$, are calculated for $k=6,8,50,100,150,200$, and 250. The last row is calculated within the framework of the continued fractions method.

\begin{tabular}{rcc}
\hline \multicolumn{1}{c}{$s=1, l=1, n=0$} & $s=2, l=2, n=0$ \\
\hline & $0.496526544-0.184975418 i$ & $0.747343368-0.17792463 i$ \\
\hline 6 & $0.4965264765276178-0.1849753180356798 i$ & $0.7473429777005388-0.1779414742208077 i$ \\
8 & $0.4965265309901696-0.1849754375379761 i$ & $0.7473517597949078-0.1779529416751695 i$ \\
50 & $0.4965265283562174-0.1849754359058844 i$ & $0.7473433688364812-0.1779246313780083 i$ \\
100 & $0.4965265283562174-0.1849754359058844 i$ & $0.7473433688360837-0.1779246313778714 i$ \\
150 & $0.4965265283562174-0.1849754359058844 i$ & $0.7473433688360837-0.1779246313778714 i$ \\
200 & $0.4965265283562174-0.1849754359058844 i$ & $0.7473433688360837-0.1779246313778714 i$ \\
250 & $0.4965265283562174-0.1849754359058844 i$ & $0.7473433688360837-0.1779246313778714 i$ \\
CF & $0.4965265283562174-0.1849754359058844 i$ & $0.7473433688360837-0.1779246313778714 i$ \\
\hline
\end{tabular}

TABLE IX: The complex frequencies of the electromagnetic (left column) and the gravitational (right column) quasinormal modes. The Padé approximants, $\mathcal{P}_{k}^{k}$, are calculated for $k=6,8,50,100,150,200$, and 250

\begin{tabular}{rcc}
\hline \multicolumn{1}{c}{$s=1, l=1, n=1$} & $s=2, l=2, n=1$ \\
\hline $0.42903084-0.587335292 i$ & $0.693421994-0.54782975 i$ \\
\hline 6 & $0.4290303098689009-0.5873349677300929 i$ & $0.6925703481161420-0.5478549212072782 i$ \\
8 & $0.4290305267101144-0.5873351193512594 i$ & $0.6931350943803045-0.5477990964454646 i$ \\
50 & $0.4290308391272459-0.5873352910914822 i$ & $0.6934219986356213-0.5478297513079539 i$ \\
100 & $0.4290308391272117-0.5873352910914573 i$ & $0.6934219937602342-0.5478297505799500 i$ \\
150 & $0.4290308391272117-0.5873352910914573 i$ & $0.6934219937583319-0.5478297505824732 i$ \\
200 & $0.4290308391272117-0.5873352910914573 i$ & $0.6934219937583269-0.5478297505824696 i$ \\
250 & $0.4290308391272117-0.5873352910914573 i$ & $0.6934219937583269-0.5478297505824696 i$
\end{tabular}

\section{B. Reissner-Nordström black hole}

We conclude this section with a brief discussion of the perturbations of the Reissner-Nordström black hole. The normal modes satisfy the differential equation (1) with the potential of the form

$$
V(x)=\left(1-\frac{1}{r}+\frac{Q^{2}}{r^{2}}\right)\left(\frac{l(l+1)}{x^{2}}-\frac{q_{2 s-3}}{x^{3}}+\frac{4 Q^{2}}{x^{4}}\right)
$$

where the parameters $q_{ \pm}$are defined as

$$
q_{ \pm 1}=\frac{1}{2}\left(3 \pm \sqrt{9+16 q^{2}(l-1)(l+2)}\right)
$$


TABLE X: The complex frequencies of the electromagnetic (left column) and the gravitational (right column) quasinormal modes. The Padé approximants, $\mathcal{P}_{k}^{k}$, are calculated for $k=6,8,50,100,150,200$, and 250

\begin{tabular}{rcc}
\hline \multicolumn{1}{c}{$s=1, l=1, n=2$} & $s=2, l=2, n=2$ \\
\hline $0.349547136-1.050375198 i$ & $0.60210691-0.956553966 i$ \\
\hline 6 & $0.3489671476223886-1.0496242138899032 i$ & $0.5983320642239965-0.9571047257361685 i$ \\
8 & $0.3495311626539206-1.0501579826696264 i$ & $0.6010669702662552-0.9565205248055362 i$ \\
50 & $0.3495471345091076-1.0503751985469316 i$ & $0.6021058209312484-0.9565564091949364 i$ \\
100 & $0.3495471352140788-1.0503751987176345 i$ & $0.6021069091277328-0.9565539641411267 i$ \\
150 & $0.3495471352140217-1.0503751987176476 i$ & $0.6021069092310929-0.9565539664324743 i$ \\
200 & $0.3495471352140216-1.0503751987176475 i$ & $0.6021069092254648-0.9565539664461968 i$ \\
250 & $0.3495471352140216-1.0503751987176475 i$ & $0.6021069092247347-0.9565539664461398 i$
\end{tabular}

TABLE XI: The complex frequencies of the electromagnetic (left column) and the gravitational (right column) quasinormal modes. The Padé approximants, $\mathcal{P}_{k}^{k}$, are calculated for $k=6,8,50,100,150,200$, and 250

\begin{tabular}{rcc}
\hline \multicolumn{1}{r}{$k$} & $s=1, l=1, n=3$ & $s=2, l=2, n=3$ \\
\hline & $0.292353398-1.543817848 i$ & $0.503009924-1.410296404 i$ \\
\hline 6 & $0.288963705877476-1.544282449993818 i$ & $0.497642737535928-1.414461228437683 i$ \\
8 & $0.291218230899165-1.542799891892294 i$ & $0.502401150125607-1.403417125878443 i$ \\
50 & $0.292353425313164-1.543817856808729 i$ & $0.503101540951305-1.410129166751418 i$ \\
100 & $0.292353398843248-1.543817848040974 i$ & $0.503010351175324-1.410295016204194 i$ \\
150 & $0.292353398833784-1.543817847996815 i$ & $0.503009929112050-1.410296418310432 i$ \\
200 & $0.292353398833987-1.543817847996173 i$ & $0.503009925121021-1.410296404044260 i$ \\
250 & $0.292353398834001-1.543817847996167 i$ & $0.503009924395821-1.410296404890974 i$ \\
\hline
\end{tabular}

and $s=1$ and $s=2$ for the electromagnetic and gravitational perturbations, respectively. It should be emphasized that all the results cited in Ref. [25] have been easily reproduced by constructing the appropriate Padé transforms of the perturbation series. Since the results of our calculations follow the common pattern we shall discuss only one exemplary configuration with $Q=1 / 5$ and $l=2$ (see Tab. XIV). First, we have found that in order to calculate the quasinormal frequency of the $(2,0)$ mode with the accuracy quoted in [25], we need $\mathcal{P}_{k}^{k}$ for $k \geq 158$. Moreover, starting with $k=206$ the result stabilizes with an accuracy of (at least) 24 decimal places. It should be noted that the Borel summation applied to $\tilde{\mathcal{P}}_{250}^{250}$ gives precisely the same result. For the higher overtones both methods give very close results. 
TABLE XII: The complex frequencies of the electromagnetic (left column) and the gravitational (right column) quasinormal modes. The Padé approximants, $\mathcal{P}_{k}^{k}$, are calculated for $k=6,8,50,100,150,200$, and 250

\begin{tabular}{rcc}
\hline \multicolumn{1}{r}{$k$} & $s=1, l=1, n=4$ & $s=2, l=2, n=4$ \\
\hline & $0.253108292-2.045100568 i$ & $0.41502916-1.893689782 \mathrm{i}$ \\
\hline 6 & $0.248727904828560-2.051639959255668 i$ & $0.419396702975745-1.887928109921840 i$ \\
8 & $0.249369705013264-2.043584186827301 i$ & $0.362047466802911-1.868264500927380 i$ \\
50 & $0.253108599503254-2.045101202339157 i$ & $0.415360773226012-1.890523875905394 i$ \\
100 & $0.253108285283085-2.045100567418578 i$ & $0.415038826730706-1.893625733917996 i$ \\
150 & $0.253108292027439-2.045100567596560 i$ & $0.415026686988849-1.893690158005340 i$ \\
200 & $0.253108291983075-2.045100567606178 i$ & $0.415029155910513-1.893689969992049 i$ \\
250 & $0.253108291980756-2.045100567606751 i$ & $0.415029158009993-1.893689749075853 i$
\end{tabular}

TABLE XIII: The complex frequencies of the electromagnetic (left column) and the gravitational (right column) quasinormal modes. The Padé approximants, $\mathcal{P}_{k}^{k}$, are calculated for $k=6,8,50,100,150,200,250,300$ and 350

\begin{tabular}{rcc}
\hline \multicolumn{1}{c}{$s$} & $s=1, l=1, n=5$ & $s=2, l=2, n=5$ \\
\hline & $0.224505582-2.547851238 i$ & $0.338598806-2.391216108 i$ \\
\hline 6 & $0.222128242094723-2.559732389855251 i$ & $0.306732391439588-2.297611394962681 i$ \\
8 & $0.217300021185279-2.546769260540304 i$ & $0.244507176638539-2.455516877615405 i$ \\
50 & $0.224508451763544-2.547853939163384 i$ & $0.310122138984459-2.392572467682007 i$ \\
100 & $0.224505537892275-2.547851205268914 i$ & $0.337381549712206-2.391221582182717 i$ \\
150 & $0.224505582024767-2.547851238125507 i$ & $0.338677506810238-2.391243385562942 i$ \\
200 & $0.224505581675340-2.547851238746054 i$ & $0.338592848394283-2.391225800860825 i$ \\
250 & $0.224505581611109-2.547851238755523 i$ & $0.338596687693972-2.391217123833891 i$ \\
300 & $0.224505581599715-2.547851238757362 i$ & $0.338598570386376-2.391216091649921 i$ \\
350 & $0.224505581599435-2.547851238756736 i$ & $0.338598780167286-2.391216068179005 i$ \\
\hline
\end{tabular}

We conclude this subsection with a comment that also relates to our previous discussion. First, it should be noted that calculation of the Padé transforms or making use of the Wynn algorithm takes only a small fraction of the total time. Most of the computation time is spent on construction of the $\Lambda_{k}$ parameters. Moreover, in order to avoid zero divisors one has to retain as many digits as necessary, that may considerably slow down the calculation. Nevertheless, they can be completed in a reasonable time. On the other hand, calculation of the integral transforms (8) may be tricky and time consuming. 
TABLE XIV: The complex frequencies of the gravitational quasinormal $l=2$ modes of the ReissnerNordström black hole. $Q=1 / 5$.

\begin{tabular}{lll}
\hline \multicolumn{3}{c}{ mode method } \\
\hline$n=0$ & $\mathcal{P}_{250}^{250}$ & $0.756873775755127489560243-0.178796227993424581484091 i$ \\
$n=0$ & $\mathcal{B}_{250}^{250}$ & $0.756873775755127489560243-0.178796227993424581484091 i$ \\
$n=1$ & $\mathcal{P}_{250}^{250}$ & $0.703455061389587189545552-0.550248607591426397610300 i$ \\
$n=1$ & $\mathcal{P}_{300}^{300}$ & $0.703455061389587189590323-0.550248607591426397588809 i$ \\
$n=1$ & $\mathcal{B}_{250}^{250}$ & $0.703455061389587189595369-0.550248607591426397589084 i$ \\
$n=2$ & $\mathcal{P}_{250}^{250}$ & $0.612848616773723964811455-0.959880899685908114593243 i$ \\
$n=2$ & $\mathcal{P}_{350}^{350}$ & $0.612848616773725485129278-0.959880899685896111782658 i$ \\
$n=2$ & $\mathcal{B}_{350}^{350}$ & $0.612848616773725500883098-0.959880899685896118184149 i$ \\
\hline
\end{tabular}

\section{FINAL REMARKS}

We have developed a simple strategy for calculating quasinormal modes of the black holes by extending the approach propounded in Paper I. The basic ingredients of the method are the generalization of the Iyer-Will technique to higher-order terms of the WKB approximation and subsequent summation of the thus obtained perturbation series with the aid of the Padé transforms. Although the general form of the functions $\Lambda_{k}$ are known only for $k \leq 16$, the higher-order $\Lambda_{k}$ parameters (for a given harmonic and overtone number) can easily be calculated numerically. Once the $\Lambda_{k}$ are known, the complex quasinormal frequency can be obtained from the Padé transform of the perturbation series. Our method has been successfully used to calculate the quasinormal modes of the Schwarzschild and Reissner- Nordström black holes. Indeed, we have demonstrated that the results are amazingly accurate, even for the overtones. For example, the fundamental gravitational $l=2$ mode calculated using the continued fractions method agrees with the result of our approach to 24 decimal places, which is really impressive. We are unable to answer the question if the present technique works well for the highly-damped modes. The answer would require calculations of the Padé transforms far beyond the limit assumed in this paper. However, all the cases that have been considered here seem to obey a simple rule: the more terms of the perturbation series are retained and transformed the better the final result is obtained, although the improvement may be slow ${ }^{3}$.

\footnotetext{
${ }^{3}$ It should be noted that the convergence of the continued fraction method is also slow.
} 
We have demonstrated that our method is accurate and useful and we believe that it is also the simplest one. It can be applied to a wide class of problems practically without any change. Indeed, its simple black-box structure, with the (expanded) effective potential as the input and the accurate quasinormal frequencies as the output is certainly its attractive feature. Moreover, it is relatively fast and all the steps are numerically stable. We believe that it also would be the first method of choice in many applications and experimentation, as summing up the terms of the perturbation series is generally a bad strategy. The Mathematica notebooks with examples and explanations can be obtained from the first author upon request.

\section{Appendix}

In this Appendix, we describe briefly the Wynn epsilon algorithm heavily used in this paper. First, let us introduce our notation. Let $S=\left(s_{0}, s_{1}, \ldots, s_{i} \in \mathbb{C}\right)$ be a sequence of the partial sums (in our case it is the sequence of the WKB approximants). The Wynn algorithm consists of the initialization phase in which we put

$$
S_{j, 0}=s_{j}
$$

and define

$$
S_{j,-1}=0
$$

and the iterative phase

$$
S_{j, k+1}=S_{j+1, k-1}+\frac{1}{S_{j+1, k}-S_{j, k}}
$$

In the Wynn $\epsilon$ algorithm we are interested in terms with even $k$. Typically, the terms with odd $k$ grow and the approximants are labeled by even $k$. A few last columns of $S_{j, k}$, where $S_{j, 0}(j=$ $\left.0,1, \ldots, n_{\max }\right)$ are the partial sums of the terms in (3) are schematically shown in Fig. 3 . There is a deep connection between the $S_{j, k}$ terms and the Padé approximants. Indeed, the entries $S_{j, 2 k}$ in the columns labeled by the even index are equal to the Padé approximants

$$
S_{j, 2 k}=P_{k}^{j+k}
$$

provided the usual assumptions of the existence of the approximants $P$ and nonexistence of the zero divisors in the construction of the $S$ transforms are satisfied. It should be noted that the remaining approximants of the Padé table should be constructed independently. Specifically, of the diagonal approximants $P_{k}^{k}$ and $P_{k+1}^{k}$ only the former can be calculated this way. 


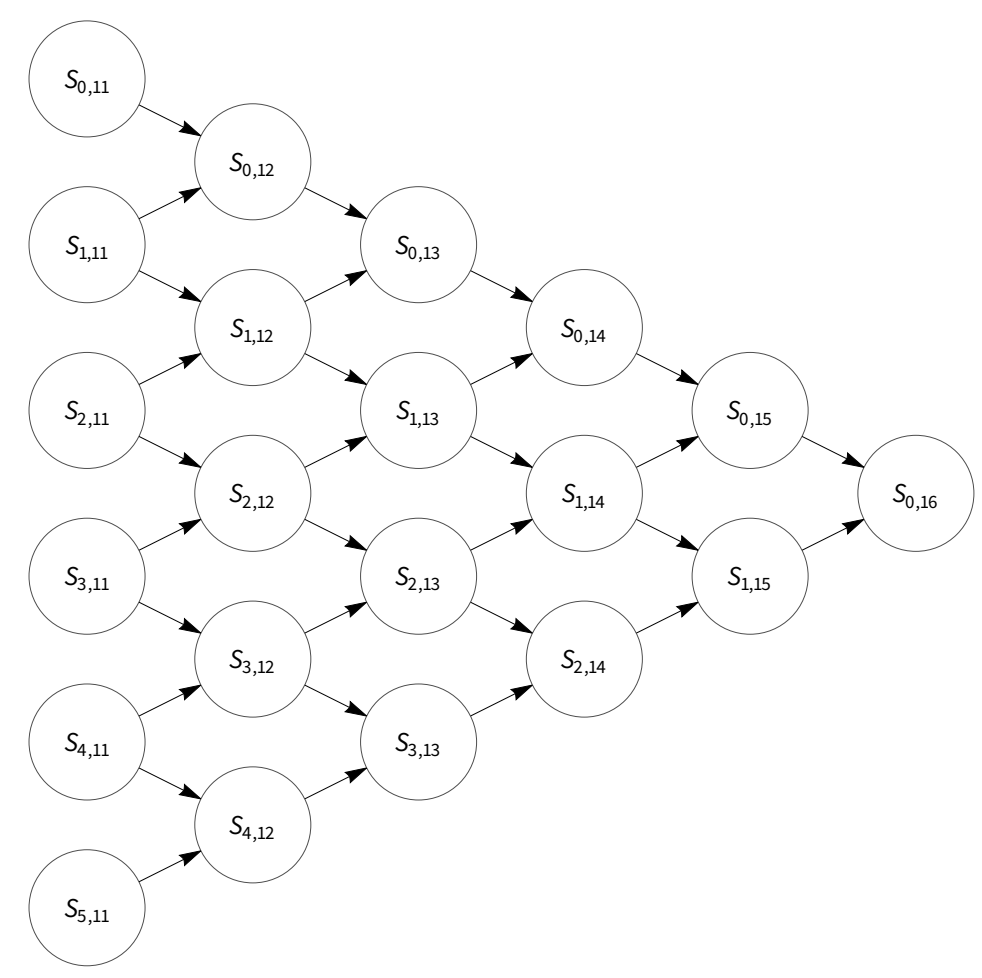

FIG. 3: The few last columns of the $\epsilon$ transforms of the 16th order problem. It should be noted that although only the last entry inherits all previous $S$-terms, each $S_{j, k}$ does inherit all $\tilde{\Lambda}_{i}$ up to $j+k$.

The Wynn algorithm is simple and very efficient, however, since the elements of the odd columns grow there is a real danger of numerical instabilities. This can be cured by retaining the sufficient number of digits in the calculations. The diamond-like structure of the Wynn algorithm makes it very easy to use.

[1] J. Matyjasek and M. Opala, Phys. Rev. D96, 024011 (2017).

[2] C. V. Vishveshwara, Phys. Rev. D1, 2870 (1970).

[3] E. W. Leaver, Proc. Roy. Soc. Lond. A402, 285 (1985).

[4] H.-P. Nollert, Phys. Rev. D47, 5253 (1993).

[5] A. Rostworowski, Acta Phys. Polon. B38, 81 (2007).

[6] H. T. Cho, A. S. Cornell, J. Doukas, and W. Naylor, Class. Quant. Grav. 27, 155004 (2010).

[7] B. Majumdar and N. Panchapakesan, Phys. Rev. D40, 2568 (1989).

[8] H.-P. Nollert and B. G. Schmidt, Phys. Rev. D45, 2617 (1992).

[9] A. Jansen, Eur. Phys. J. Plus 132, 546 (2017).

[10] V. Cardoso, M. Kimura, A. Maselli, E. Berti, C. F. B. Macedo, and R. McManus, Phys. Rev. D99, 
104077 (2019).

[11] R. McManus, E. Berti, C. F. B. Macedo, M. Kimura, A. Maselli, and V. Cardoso Parametrized black hole quasinormal ringdown. II. Coupled equations and quadratic corrections for nonrotating black holes (2019), ArXiv:1906.05155.

[12] B. F. Schutz and C. M. Will, Astrophys. J. 291, L33 (1985).

[13] S. Iyer and C. M. Will, Phys. Rev. D35, 3621 (1987).

[14] S. Iyer, Phys. Rev. D35, 3632 (1987).

[15] K. D. Kokkotas and B. F. Schutz, Phys. Rev. D37, 3378 (1988).

[16] E. Seidel and S. Iyer, Phys. Rev. D41, 374 (1990).

[17] N. Froeman, P. O. Froeman, N. Andersson, and A. Hoekback, Phys. Rev. D45, 2609 (1992).

[18] N. Andersson and S. Linneaus, Phys. Rev. D46, 4179 (1992).

[19] O. B. Zaslavskii, Phys. Rev. D43, 605 (1991).

[20] D. V. Gal'tsov and A. A. Matiukhin, Class. Quant. Grav. 9, 2039 (1992).

[21] H. Blome and B. Mashhoon, Physics Letters A 100 (1984).

[22] V. Ferrari and B. Mashhoon, Phys. Rev. Lett. 52, 1361 (1984).

[23] V. Ferrari and B. Mashhoon, Phys. Rev. D30, 295 (1984).

[24] R. A. Konoplya, Physical Review D 68, 024018 (2003).

[25] Y. Hatsuda Quasinormal modes of black holes and Borel summation (2019), ArXiv:1906.07232.

[26] T. Sulejmanpasic and M. Ünsal, Comput. Phys. Commun. 228, 273 (2018), 1608.08256.

[27] C. M. Bender and T. T. Wu, Phys. Rev. D7, 1620 (1973).

[28] C. M. Bender and S. A. Orszag, Advanced mathematical methods for scientists and engineers (McGrawHill, New York, 1978).

[29] P. Wynn, Mathematical Tables and Other Aids to Computation 10, 91 (1956).

[30] C. Brezinski and M. R. Zaglia, Extrapolation methods: theory and practice (Elsevier, Amsterdam, 1991).

[31] L. D. Landau and E. M. Lifshitz, Quantum mechanics: non-relativistic theory (in Russian) (Nauka, Moscow, 1989).

[32] N. Andersson, Proc. R. Soc. 439, 47 (1992). 